compare the long-term survival of patients with obesity hypoventilation syndrome (OHS), COPD and overlap syndrome who were established on NIV.

Methods All patients with a diagnosis of COPD, OHS and overlap syndrome were identified retrospectively from a patient database. Overlap syndrome was defined as COPD and either OHS or obstructive sleep apnoea resulting in chronic type 2 respiratory failure. The diagnosis was defined at the time NIV was established from medical assessment and respiratory physiology. All patient data was anonymised. A Kaplan-Meier survival analysis was performed. Median survival was estimated for each of the three groups. Survival was compared using Mantel-Cox test, Gehan-Breslow-Wilcoxon test and Log-rank test.

Results In total 463 patients were established on NIV. NIV was initiated on 158 patients with COPD (51\% female, 49\% male, mean age at set up 66 years), 269 patients with OHS (46\% female, 54\% male, mean age 62 years) and 36 patients with overlap syndrome (48\% female, $52 \%$ male, mean age 66 years). The Kaplan-Meier survival curves for the three groups are shown. A clinically and statistically significant difference in survival was observed between the three groups $(p<0.0001)$. Patients with COPD had the worst long term survival compared with patients with OHS and the overlap syndrome. The median survival was 49 months for patients with COPD, 92 months for patients with overlap syndrome and 141 for patients with OHS. Conclusion Evidence for domiciliary NIV in patients with OHS is well established. There is emerging evidence to support with use of NIV in patients with chronic hypercapnic COPD and low body mass index. Patients with overlap syndrome are a heterogeneous group representing a spectrum from predominately COPD to predominately OHS. Further studies are required to establish if patients with overlap syndrome benefit from NIV and to identify potentially modifiable risk factors associated with a poor outcome.

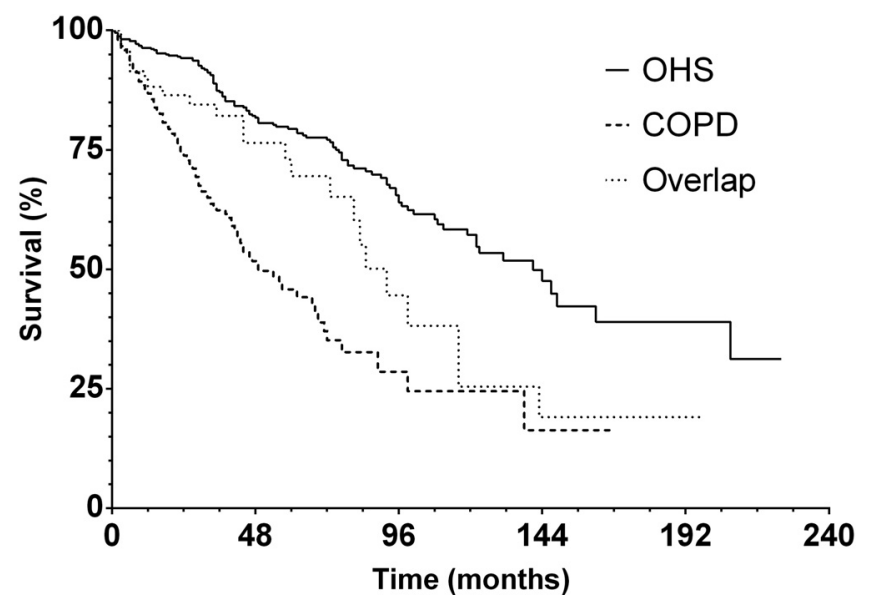

Abstract P191 Figure 1 Showing the survival for patients with obesity hypoventilation syndrome (OHS), COPD and overlap syndrome

\section{P192 CLINICAL EFFECTIVENESS OF NON-INVASIVE VENTILATION IN PATIENTS WITH MOTOR NEURON DISEASE}

D Freeman, A Jothieswaran, M Mascareno, N Chaudhry, S Bokhari, TW Felton, AM Bentley. University Hospital of South Manchester, Manchester, UK

10.1136/thoraxjnl-2015-207770.329
Introduction and objectives The use of long term non-invasive ventilation (NIV) for type II respiratory failure caused by Motor Neuron Disease (MND) is well recognised. In patients with MND and good bulbar function, NIV has been shown to improve survival and quality of life. ${ }^{1}$ NIV use in patients with MND has increased since the publication of the National institute of Clinical Health Excellence guidelines in July 2010. Our objective was to identify the clinical effectiveness of NIV in all patients with MND referred to a large tertiary referral teaching hospital service.

Methods All patients with MND established on NIV were identified retrospectively from a clinical database. Patients were excluded from the analysis if there was another diagnosis as the main indication for establishing NIV. All patient data was anonymised. A Kaplan-Meier survival analysis was performed and the median survival was estimated.

Results 222 patients with MND established on NIV were identified. The median age was 64 years (range 19-90 years). One hundred and forty patients (63\%) were male and $82(37 \%)$ female. The median survival was 436 days on NIV. The KaplanMeier survival curve is shown below.

Conclusion A median survival of 436 days compares favourably with the median survival of 219 days identified in patients with MND receiving NIV in the trial by Bourke et al. Our patient cohort included patients with both limb onset and bulbar onset forms of the disease. The impact on survival may, in part, be due to NIV but overall improvements in medical care, supporting adequate nutrition and assisted cough techniques in a specialist centre will have contributed.

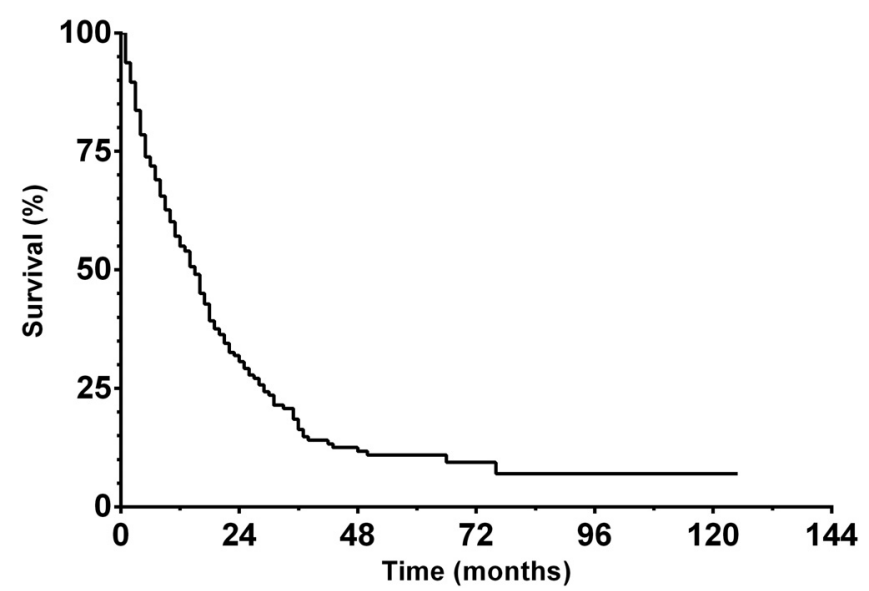

Abstract P192 Figure 1 Showing the survival of patients with motor neurone disease receiving NIV

\section{REFERENCE}

1 Bourke SC, et al. Effects of non-invasive ventilation on survival and quality of life in patients with amyotrophic lateral sclerosis: a randomised controlled trial. Lancet Neurol. 2006;5:140-7

\section{P193 HOW SAFE IS DOMICILIARY CHANGE OF TRACHEOSTOMY TUBE IN VENTILATOR DEPENDENT PATIENTS?}

JM Palmer. Plymouth Hospitals NHS Trust, Plymouth, UK

\subsection{6/thoraxjn-2015-207770.330}

Introduction Tracheostomy ventilation (T-HMV) is indicated in a small group of patients with chronic ventilatory failure. These 
patients often reside outside of formal health-care environments. Tracheostomy tubes generally need to be changed monthly. Our unit undertakes the majority of tube changes in the patient's home. There are little data evaluating the safety of this procedure outside of the hospital.

Method We conducted a retrospective review of domiciliary tracheostomy tube changes on ventilator dependent patients. Concurrently all HMV-UK network centres were sent a basic electronic survey. Data collection took place during December 2014.

Results E-Surveys were sent to 37 centres. Responses were received from $12(32 \%)$. 75\% $(\mathrm{n}=9)$ of those responding undertake the majority of tracheostomy changes in the community, 1 centre brings patients into hospital. 2 others do not routinely manage T-HMV patients. Tube changes undertaken at home, are frequently but not exclusively completed by trained professionals including care support workers. 5 areas reported that family members undertake some domiciliary tube changes.

The notes of 11 ventilator dependent T-HMV patients were reviewed. Each patient had a mean 9.2 domiciliary tube changes undertaken by the respiratory outreach team. $72 \%(\mathrm{n}=66)$ of changes took place without complication or incident. Of the 26 changes which had documented complications, $69 \%$ related to minor bleeding only, 3 described moderate bleeding. 5 changes were associated with incidents. 3 of these related to difficulty inserting a new tube with 1 patient requiring a smaller diameter replacement tube. 1 patient, erroneously, had a wrong diameter tube inserted, this was not replaced as the patient found it more comfortable and continued to ventilate effectively. 1 change was associated with loss of speech for 24-hours post procedure. Nobody was admitted or harmed as a direct result of a tube changed at home.

The notes of a further patient were reviewed. Approximately 50 domiciliary tube changes were undertaken by her brother without supervision or involvement of health care workers. There were no documented complications or admissions as a result of these changes.

Conclusion Domiciliary tracheostomy tube change by trained personnel on ventilator dependent patients is safe and effective.

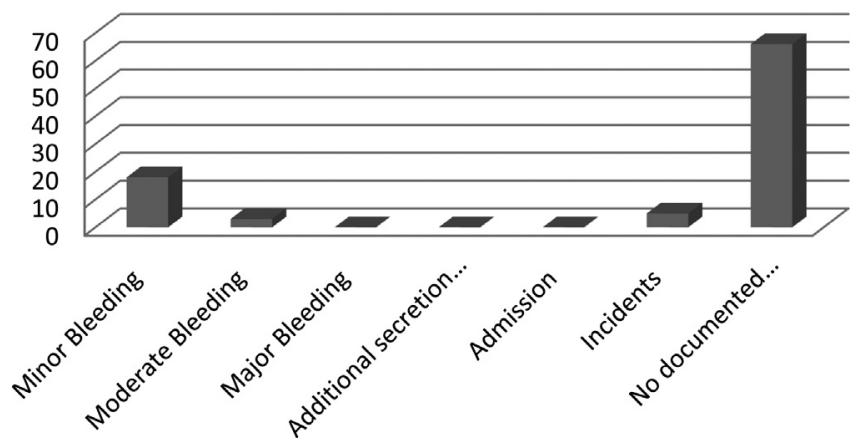

Abstract P193 Figure 1 Chart detailing the outcome of 92 domiciliary tracheostomy changes on ventilator dependent patients

\section{P194 INPATIENT ADJUSTMENT OF SUB-OPTIMAL HOME MECHANICAL VENTILATION (HMV) - AN EFFECTIVE USE OF RESOURCES?}

F Frost, B Al-Hakim, S Wordingham-Baker, V Ford, H Ashcroft, K Ward, R Parker, B Chakrabarti, R Angus, N Duffy. Ventilation Inpatient Centre, Aintree University Hospital, Liverpool, UK
Introduction HMV can be initiated and monitored as either inpatient or outpatient. There is little evidence for best practice in this field and inpatient ventilation beds are a scarce resource. We evaluated patients, with sub-optimal HMV, admitted to our tertiary unit for adjustments to consider whether these admissions were successful, and hence an effective use of resources.

Methods Patients were identified from our ventilation unit's database. Notes, oximetry and ventilator download from preadmission, pre-discharge and post-discharge were retrospectively analysed.

Results In a 6-month period (June-December 2013) 30 patients were admitted to our unit for adjustments of HMV. 43\% were female. Obesity related sleep disorder formed the majority of underlying conditions (53\%), with musculoskeletal deformities $(20 \%)$ and neuromuscular conditions (10\%) also frequently seen. Median length of stay was 2 days. HMV was discontinued during admission in 2 cases in line with patient wishes.

$19(63 \%)$ were deemed to have had successful admissions, defined as normalisation of at least one abnormal ventilation parameter $\left(\mathrm{pCO}_{2}>6.0\right.$, desaturations $>14 / \mathrm{hr}$, time below $90 \%$ of $>30 \mathrm{~min}$, mean saturations of $<88 \%$, usage $>6 \mathrm{hrs}$, leak $<50 \mathrm{~L} / \mathrm{min}$ ). Of the 19 successful admissions, 6 showed sustained improvement post-discharge. 11 (37\%) admissions were deemed unsuccessful, poor baseline usage and missed outpatient appointments were observed in this group. Noteworthy improvements were made to oximetry parameters during admission, although not all of these were maintained post-discharge (Table 1).

\begin{tabular}{llll}
$\begin{array}{l}\text { Abstract P194 Table } 1 \\
\mathrm{~N}=30\end{array}$ & \multicolumn{3}{c}{ Oximetry } \\
\cline { 2 - 4 } & Desaturations $>\mathbf{1 4 / h r}$ & $>30$ mins $<90 \%$ & Mean saturations $<88 \%$ \\
\hline Pre admission & $8(27 \%)$ & $23(77 \%)$ & $9(30 \%)$ \\
Pre discharge & $2(7 \%)$ & $6(20 \%)$ & $3(10 \%)$ \\
Post discharge & $4(13 \%)$ & $18(60 \%)$ & $2(7 \%)$ \\
\hline
\end{tabular}

Ventilator leak and usage information was available for 22 (73\%) patients. Excess leak $(50>\mathrm{L} / \mathrm{min})$ was seen in 10 patients pre-admission, only 1 patient had excess leak post-discharge. Pre-admission usage of $<2 \mathrm{~h} /$ night was seen in 6 patients, only 1 showed sustained improvement in usage. 8 patients were admitted with usage of 2-4 h, 4 improved post discharge usage to $>6 \mathrm{~h}$ and only 1 showed deterioration in usage.

Conclusion Admitting patients for adjustments to HMV can improve ventilation parameters yet only some of these improvements are maintained after discharge. There appears to be a subset of patients who do not benefit from inpatient admissions, particularly patients with poor baseline usage. We suggest careful selection of patients to ensure effective use of limited resources.

\section{P195 DEMOGRAPHICS AND OUTCOMES OF NIV IN MND: A FRONTLINE PERSPECTIVE}

K Ward, V Ford, H Ashcroft, S Wordingham-Baker, B Chakrabarti, N Duffy, R Angus, R Parker. Liverpool Centre for Sleep and Ventilation, Liverpool, UK

10.1136/thoraxjnl-2015-207770.332 